\title{
CONSTRUÇÃO DA FORMA MATRICIAL DE SEQUÊNCIAS LINEARES E RECORRENTES: UM ESTUDO DA MATRIZ GERADORA
}

\author{
CONSTRUCTION OF THE MATRIAL FORM OF LINEAR AND RECURRENT \\ SEQUENCES: A STUDY OF THE GENERATING MATRIX
}

\author{
RENATA P. M. VIEIRA ${ }^{a}$ \\ FRANCISCO R. V. ALVES ${ }^{b}$ \\ PAULA M. M. C. CATARINO ${ }^{\mathrm{c}}$ \\ ANABELA M. F. B. V. RODRIGUES ${ }^{d}$
}

\begin{abstract}
Resumo
O presente artigo tem como objetivo, apresentar um caminho para o ensino da construção da forma matricial de sequências lineares e recorrentes, bem como determinar a quantidade de matrizes válidas para cada sequência e, como essas são obtidas. Utilizando a sequência de Padovan como referência para esse estudo, e a metodologia de ensino baseada na Teoria das Situações Didáticas, apresentamos uma proposta de atividade, com o viés de atingir o objetivo dessa pesquisa. Em muitos trabalhos encontrados na literatura, essas matrizes são apenas introduzidas, não sendo mostrado o seu processo de construção. Com isso, faz-se necessário, que esse estudo seja realizado, facilitando a obtenção de outras sequências, visto que nessa área, estão sendo introduzidas, cada vez mais, novas sequências em artigos de matemática pura e na área de ensino.

Palavras-chave: Forma matricial, sequências lineares, Teoria das Situações Didáticas.
\end{abstract}

\begin{abstract}
This article aims to present a path for teaching the construction of the matrix form of linear and recurrent sequences, as well as determining the number of valid matrices for each sequence and how they are obtained. Using the Padovan sequence as a reference for this study, and the teaching methodology based on the Theory of Didactic Situations, we present an activity proposal, with the aim of achieving the objective of this research. In many works found in the literature, these matrices are only introduced, and their construction process is not shown. With that, it is necessary, that this study is carried out, facilitating the obtaining of other sequences, since in this area, new sequences are being introduced, more and more, in pure mathematics articles and in the teaching area.
\end{abstract}

Keywords: Matrix form, linear sequences, Theory of Didactic Situations.

MSC2010: 11B37, 11B39

\footnotetext{
${ }^{a}$ Secretaria de Educação do Estado do Ceará, Fortaleza - Brasil; ORCID: 0000-0002-1966-7097 E-mail: re.passosm@gmail.com

${ }^{\mathrm{b}}$ Instituto Federal de Educação, Ciência e Tecnologia do Estado do Ceará, Fortaleza - Brasil; ORCID: 0000-0003-

3710-1561 E-mail: fregis@gmx.fr

${ }^{c}$ Universidade de Trás-os-Montes e Alto Douro, Vila Real - Portugal; ORCID: 0000-0001-6917-5093 E-mail: pcatarino23@gmail.com

${ }^{\mathrm{d}}$ Universidade de Trás-os-Montes e Alto Douro, Vila Real - Portugal; ORCID: 0000-0002-6166-8245 E-mail:

aborges@utad.pt
} 


\section{Introdução}

Estudos referentes a sequências lineares e recorrentes estão tendo mais publicações na literatura, sendo a Sequência de Fibonacci a mais conhecida e estudada, como em trabalhos de Oliveira e Alves [6], Santos e Alves [8] e Oliveira, Alves e Silva [7], dentre outros. Essas pesquisas transformam os referidos conteúdos de matemática pura num conteúdo a ser ensinado, tratando da sua parte histórica e evolutiva.

Além disso, percebe-se ainda a existência de outras sequências, tais como a Sequência de Lucas, Jacobsthal, Pell, Padovan, Narayana e etc, seguindo uma espécie de padrão de estudo onde é necessário estudar determinados assuntos matemáticos, como: a sua fórmula de recorrência, equação característica, fórmula de Binet, função geradora e forma matricial. Os referidos conteúdos são encontrados em artigos de matemática pura, e em alguns casos, não descrevendo assim a forma como foram encontrados, mostrando apenas a sua demonstração para validar tal teorema.

A utilização de uma matriz geradora permite obter os termos de uma sequência recorrente sem necessitar conhecer os seus termos anteriores, obtendo assim a sua forma matricial. Porém, esse conteúdo é encontrado somente em artigos de matemática pura, não ficando clara a forma como essa matriz foi construída.

Com isso, justifica-se essa pesquisa norteada pela seguinte questão: como obter a forma matricial de uma sequência linear e recorrente? Dessa forma, em torno da pergunta norteadora, pode-se traçar o objetivo geral dessa pesquisa como sendo realizar o ensino da forma matricial de uma sequência linear e recorrente, baseada em estudos investigativos em torno da sequência de Padovan.

Visando favorecer o cenário de ensino, utiliza-se como metodologia de ensino a Teoria das Situações Didáticas, elaborando situações-problema instigando os discentes em compreender o ensino da forma matricial de sequências lineares e recorrentes, através de investigação de conceitos matemáticos baseados na sequência de Padovan.

Doravante, será discutida a metodologia de ensino Teoria das Situações Didáticas de Brousseau [4], abordando as suas situações de acordo com um levantamento bibliográfico. Em seguida será analisada a forma matricial de uma sequência recorrente, sendo então selecionada a Sequência de Padovan, finalizando com uma atividade proposta visando realizar o ensino da forma matricial de sequências lineares e recorrentes. 


\section{Teoria das Situações Didáticas}

A fim de realizar uma investigação em sala de aula, utilizou-se nessa pesquisa a metodologia de ensino fundamentada na Teoria das Situações Didáticas, dando um destaque ao estudante. Essa metodologia realiza um trabalho de modelagem indicando como um determinado conteúdo matemático poderá ser exposto para um discente, objetivando alcançar o seu processo de ensino e aprendizagem. Segundo Teixeira e Passos [13], essa teoria tem como meta principal caracterizar o processo de aprendizagem através de uma série de situações reprodutíveis, chamadas de situações didáticas, elencando assim alguns fatores determinísticos para a evolução dos comportamentos dos alunos.

Vieira e Alves [14], destacam ainda que essa teoria:

é um modelo teórico, segundo o qual, considerando o ensino como projeto e
ação social em que o aprendiz se apropria de um saber constituído ou em
constituição, a didática da matemática se transforma numa ciência das
condições de transmissão e apropriação dos conhecimentos matemáticos.

Assim, pode-se destacar que o objeto de estudo dessa metodologia é a situação didática. Durante esse estudo, existem interações entre a tríade composta pelo professor, aluno e o saber, destacando os erros dos discentes durante essa troca de informações, uma vez que o aprendizado também é realizado diante de um erro discutido. Almouloud [1] apresenta uma situação didática como sendo dividida em quatro hipóteses:

1-O aluno aprende adaptando-se a um Milieu que é fator de dificuldades, de contradições [...] 2-O Milieu não munido de intenções didática é insuficiente para permitir a aquisição de um conhecimento matemático pelo aprendiz [...] 3-Esse Milieu e essas situações devem engajar fortemente os saberes matemáticos envolvidos no processo de ensino e aprendizagem. 4-No fundo, o ato de conhecer dá-se conta um conhecimento anterior, destruindo conhecimentos mal estabelecidos, superando o que, no próprio espírito, é obstáculo à espiritualização.

As condições sociais apresentam dificuldades e desafios no dia a dia, muitas de natureza matemática (fazer um pagamento, saber dar um troco de uma venda e outras). Essas dificuldades serão utilizadas na formulação das metodologias de ensino que têm como ponto de partida situações concretas, até atingir os conceitos abstratos matemáticos. Assim, a presença do milieu é considerada durante todo o processo de aprendizagem, sendo um fator externo relacionado ao estudante que opta por querer aprender. Essas situações didáticas favorecem o saber, tendo uma série de fatores dependentes. Contudo, pode-se concluir que uma situação didática é o jogo de interação entre o discente e as atividades propostas pelo docente, com o objetivo de realizar o desenvolvimento autônomo do estudante. 
Existem ainda as situações a-didáticas, que são aquelas que não foram planejadas, aparecendo devido a um certo problema encontrado no decorrer do processo. Nessas situações, o professor e o aluno irão se adaptar às condições de resolução do novo problema. O aluno levará em consideração os seus conhecimentos anteriores, para tentar resolver o problema sem que, inicialmente, haja intervenção do professor. O docente deverá ter um domínio completo do conteúdo, para que possa induzir o raciocínio do aluno, de acordo com o seu nível de conhecimento, de modo que o aluno consiga, por ele próprio, chegar à solução do problema. Logo, a situação adidática e didática agem em conjunto de forma harmônica [9].

Durante as situações, podem ocorrer vários problemas, onde o professor pode realizar uma análise de acordo com a Teoria das Situações Didáticas, observando as relações ocorridas entre a tríade durante a resolução e construção da atividade proposta. Assim, essa teoria é dividida em quatro fases: ação, formulação, validação e institucionalização.

Na fase da ação, segundo Teixeira e Passos [13]:

situação didática de ação: o aluno reflete e simula tentativas, ao eleger um procedimento de resolução dentro de um esquema de adaptação, por intermédio da interação com o milieu, tomando as decisões que faltam para organizar a resolução do problema.

É nessa fase que os estudantes irão se deparar com a atividade proposta, e tentar formular ideias para a resolução do problema, apropriando-se de conhecimentos prévios existentes. É interessante o professor analisar a troca de informações com os outros alunos participantes, para identificar as dificuldades encontras no decorrer desta situação.

Na fase de formulação:

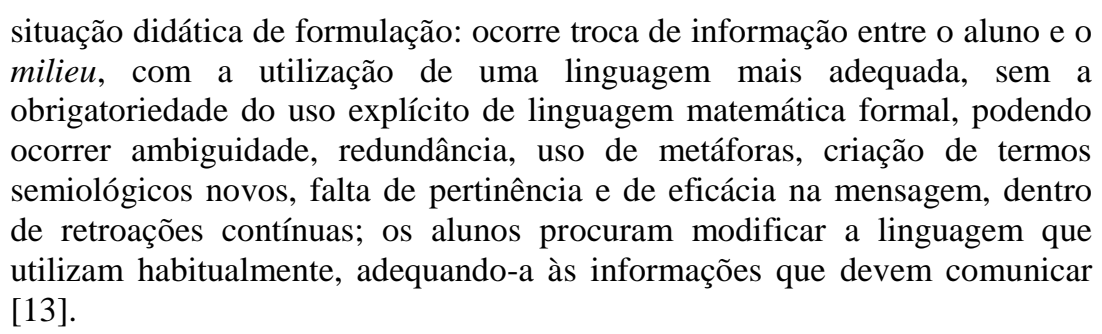

Durante essa fase, a partir das ideias geradas na etapa anterior, os estudantes irão amadurecer esse raciocínio, transformando a linguagem numa mais adequada, tornando o processo mais matemático. Com isso, será possível realizar a fase seguinte, dando continuidade à essa teoria.

$\mathrm{Na}$ fase de validação: 
situação didática de validação: os alunos tentam convencer os interlocutores da veracidade das afirmações, utilizando uma linguagem matemática apropriada (demonstrações); as situações de devolução, ação, formulação e validação caracterizam a situação adidática, em que o professor permite ao aluno trilhar os caminhos da descoberta, sem revelar sua intenção didática, tendo somente o papel de mediador [13].

Assim, os estudantes irão demonstrar os teoremas e propriedades encontrados, conjecturando as suas formulações realizadas na fase anterior. É importante analisar o erro que possa ser encontrado nessa etapa, visto que poderá ser o erro dos outros estudantes, realizando um aprendizado mútuo.

$\mathrm{Na}$ fase de institucionalização, o professor volta a entrar em ação, revelando a real intenção da atividade proposta, além de discutir os fatos ocorridos nas fases anteriores. Contudo, o ensino e aprendizagem em matemática é visto em muitos casos simplesmente pela resolução de problemas, não destacando o lado investigativo dos estudantes. Todavia, utilizando essa metodologia de ensino, pode-se perceber a presença de novas regras e situações imprevisíveis, ocasionando discussões e análises da atividade proposta, para que ocorra uma contribuição para esse processo de ensino em matemática.

\section{Representação matricial da sequência de Padovan}

Nesta seção, tomaremos como base um estudo referente à Sequência de Padovan e a sua forma matricial, para que então seja elaborada a atividade proposta na seção seguinte.

A Sequência de Padovan é uma sequência do tipo linear e recorrente, sendo de terceira ordem, criada pelo arquiteto italiano Richard Padovan (1935 - ), nascido na cidade de Pádua [12]. Esses números não possuem uma situação problema inicial, como acontece para a sequência de Fibonacci, porém, a sua criação deu-se a partir da destruição de igrejas após a Segunda Guerra Mundial. Assim, Hans Van der Laan e o seu irmão, acabaram por reconstruir essas igrejas e descobriram um novo padrão de medida, um número irracional, conhecido como número plástico.

Todavia, Padovan criou uma sequência, em que se utiliza esse número plástico, tendo o seu valor aproximadamente 1,32. Logo, os números de Padovan possuem uma equação característica, apresentando três raízes, em que a raiz real é dada pelo número plástico e as demais raízes são complexas e conjugadas [15]. Assim, esses números são definidos como:

Definição 1. A recorrência da Sequência de Padovan é dada por: 


$$
P_{n}=P_{n-2}+P_{n-3}, n \geq 3,
$$

em que $P_{n}$ é o $n$-ésimo termo da sequência e $P_{0}=P_{1}=P_{2}=1$.

O polinômio característico dessa sequência é dado pela equação $x^{3}-x-1=0$, possuindo duas raízes complexas e conjugadas e uma raiz real, denotada por $\psi$, em que:

$$
\psi=\sqrt[3]{\frac{1}{2}+\sqrt{\frac{1}{4}-\frac{1}{27}}}+\sqrt[3]{\frac{1}{2}-\sqrt{\frac{1}{4}-\frac{1}{27}}}
$$

A raiz $\psi$ é um número irracional, cujo valor aproximado é 1,32471.

A sequência de Padovan apresenta seis formas matriciais, com base nos trabalhos de e Seenukul et al. [10] e Sokhuma [11], onde são apenas apresentadas as matrizes geradoras, de ordem três por três, e validando os teoremas propostos, bem como algumas identidades discutidas no decorrer do trabalho com os valores iniciais alterados para $P_{0}=P_{1}=0, P_{2}=1$. por N.

Nos teoremas que seguem abaixo, denotamos o conjunto dos números naturais

Teorema 1. Para $Q=\left[\begin{array}{lll}0 & 1 & 0 \\ 1 & 0 & 1 \\ 1 & 0 & 0\end{array}\right]$, tem-se que $Q^{n}=\left[\begin{array}{lll}P_{n-2} & P_{n-3} & P_{n-4} \\ P_{n-1} & P_{n-2} & P_{n-3} \\ P_{n-3} & P_{n-4} & P_{n-5}\end{array}\right]$ para todo $n \in \mathrm{N}, n \geq 5$.

Demonstração. Utilizando o princípio da indução finita, temos que:

Para $n=5$, temos que:

$$
Q^{5}=\left[\begin{array}{lll}
0 & 1 & 0 \\
1 & 0 & 1 \\
1 & 0 & 0
\end{array}\right]^{5}=\left[\begin{array}{lll}
2 & 1 & 1 \\
2 & 2 & 1 \\
1 & 1 & 1
\end{array}\right]=\left[\begin{array}{lll}
P_{3} & P_{2} & P_{1} \\
P_{4} & P_{3} & P_{2} \\
P_{2} & P_{1} & P_{0}
\end{array}\right]
$$

Assim, a igualdade é válida.

Supondo que seja válido para qualquer $n=k, k \in \mathrm{N}$, temos que:

$$
Q^{k}=\left[\begin{array}{lll}
0 & 1 & 0 \\
1 & 0 & 1 \\
1 & 0 & 0
\end{array}\right]^{k}=\left[\begin{array}{lll}
P_{k-2} & P_{k-3} & P_{k-4} \\
P_{k-1} & P_{k-2} & P_{k-3} \\
P_{k-3} & P_{k-4} & P_{k-5}
\end{array}\right] \text {. }
$$

Agora, verificando que seja válido para $n=k+1$, temos que: 


$$
\begin{aligned}
& Q^{k+1}=Q^{k} Q=\left[\begin{array}{lll}
0 & 1 & 0 \\
1 & 0 & 1 \\
1 & 0 & 0
\end{array}\right]^{k}\left[\begin{array}{lll}
0 & 1 & 0 \\
1 & 0 & 1 \\
1 & 0 & 0
\end{array}\right]=\left[\begin{array}{ccc}
P_{k-2} & P_{k-3} & P_{k-4} \\
P_{k-1} & P_{k-2} & P_{k-3} \\
P_{k-3} & P_{k-4} & P_{k-5}
\end{array}\right]\left[\begin{array}{lll}
0 & 1 & 0 \\
1 & 0 & 1 \\
1 & 0 & 0
\end{array}\right] \\
& Q^{k+1}=\left[\begin{array}{lll}
P_{k-3}+P_{k-4} & P_{k-2} & P_{k-3} \\
P_{k-2}+P_{k-3} & P_{k-1} & P_{k-2} \\
P_{k-4}+P_{k-5} & P_{k-3} & P_{k-4}
\end{array}\right]=\left[\begin{array}{ccc}
P_{k-1} & P_{k-2} & P_{k-3} \\
P_{k} & P_{k-1} & P_{k-2} \\
P_{k-2} & P_{k-3} & P_{k-4}
\end{array}\right]
\end{aligned}
$$

Teorema 2. Para $Q=\left[\begin{array}{lll}0 & 1 & 0 \\ 0 & 0 & 1 \\ 1 & 1 & 0\end{array}\right]$, tem-se que $Q^{n}=\left[\begin{array}{ccc}P_{n-5} & P_{n-3} & P_{n-4} \\ P_{n-4} & P_{n-2} & P_{n-3} \\ P_{n-3} & P_{n-1} & P_{n-2}\end{array}\right]$

para todo para todo $n \in \mathrm{N}, n \geq 5$.

Demonstração. De modo análogo à demonstração realizada no Teorema 1, pode-se validar o presente teorema.

Teorema 3. Para $Q=\left[\begin{array}{lll}0 & 0 & 1 \\ 1 & 0 & 0 \\ 1 & 1 & 0\end{array}\right]$, tem-se que $Q^{n}=\left[\begin{array}{ccc}P_{n-2} & P_{n-4} & P_{n-3} \\ P_{n-3} & P_{n-5} & P_{n-4} \\ P_{n-1} & P_{n-3} & P_{n-2}\end{array}\right]$

para todo para todo $n \in \mathrm{N}, n \geq 5$.

Demonstração. De modo análogo à demonstração realizada no Teorema 1, pode-se validar o presente teorema.

Teorema 4. Para $Q=\left[\begin{array}{lll}0 & 0 & 1 \\ 1 & 0 & 1 \\ 0 & 1 & 0\end{array}\right]$, tem-se que $Q^{n}=\left[\begin{array}{ccc}P_{n-5} & P_{n-4} & P_{n-3} \\ P_{n-3} & P_{n-2} & P_{n-1} \\ P_{n-4} & P_{n-3} & P_{n-2}\end{array}\right]$

para todo para todo $n \in \mathrm{N}, n \geq 5$.

Demonstração. De modo análogo à demonstração realizada no Teorema 1, pode-se validar o presente teorema.

Teorema 5. Para $Q=\left[\begin{array}{lll}0 & 1 & 1 \\ 1 & 0 & 0 \\ 0 & 1 & 0\end{array}\right]$, tem-se que $Q^{n}=\left[\begin{array}{lll}P_{n-2} & P_{n-1} & P_{n-3} \\ P_{n-3} & P_{n-2} & P_{n-4} \\ P_{n-4} & P_{n-3} & P_{n-5}\end{array}\right]$

para todo para todo $n \in \mathrm{N}, n \geq 5$.

Demonstração. De modo análogo à demonstração realizada no Teorema 1, pode-se validar o presente teorema. 
Teorema 6. Para $Q=\left[\begin{array}{lll}0 & 1 & 1 \\ 0 & 0 & 1 \\ 1 & 0 & 0\end{array}\right]$, tem-se que $Q^{n}=\left[\begin{array}{lll}P_{n-2} & P_{n-3} & P_{n-1} \\ P_{n-4} & P_{n-5} & P_{n-3} \\ P_{n-3} & P_{n-4} & P_{n-2}\end{array}\right]$ para todo para todo $n \in \mathrm{N}, n \geq 5$.

Demonstração. De modo análogo à demonstração realizada no Teorema 1, pode-se validar o presente teorema.

\section{Metodologia para a obtenção da forma matricial de uma dada sequência}

A atividade proposta foi baseada na Teoria das Situações Didáticas, seguindo os pressupostos de Brousseau [5], destacando as principais atividades voltadas ao ensino de matemática, mais especificamente na área de sequências, sendo classificadas em situações de ação, formulação, validação e institucionalização [13].

A seguir apresentaremos duas situações-problema proposta com o objetivo de realizar o ensino da forma matricial de sequências lineares e recorrentes, baseada nas matrizes geradora de Padovan, analisando o seu processo de construção e a quantidade de matrizes válidas.

Situação-problema 1: Diante da fórmula de recorrência da sequência de Padovan $P_{n}=P_{n-2}+P_{n-3}, n \geq 3$ e da matriz geradora de Padovan, denominada de matriz base, onde:

Para $Q=\left[\begin{array}{lll}0 & 1 & 0 \\ 1 & 0 & 1 \\ 1 & 0 & 0\end{array}\right]$, tem-se que $Q^{n}=\left[\begin{array}{ccc}P_{n-2} & P_{n-3} & P_{n-4} \\ P_{n-1} & P_{n-2} & P_{n-3} \\ P_{n-3} & P_{n-4} & P_{n-5}\end{array}\right]$ para todo $n \geq 5$, com $P_{0}=P_{1}=0, P_{2}=1$ [11]. Como podemos estabelecer uma relação entre a matriz geradora $Q$ e a fórmula de recorrência $P_{n}=P_{n-2}+P_{n-3}, n \geq 3$ de Padovan?

Situação de ação: Nesta fase, os alunos tomando conhecimento do problema, irão buscar em seus conhecimentos os elementos necessários para a resolução da atividade, interagindo com os outros estudantes. Logo, eles deverão analisar a primeira coluna da matriz base dada e observar a sua regra de construção.

Situação de formulação: Os estudantes deverão perceber que na primeira coluna da matriz base estão os coeficientes existentes na fórmula de recorrência, estabelecendo uma regra para o operador, carregando consigo os valores dos coeficientes da recorrência de uma sequência. Assim, ao elevar essa matriz à $n$-ésima potência, obtêm- 
se o termo $a_{11}$ de acordo com a potência elevada e os demais termos da primeira linha são dados pelos termos sucessores da sequência linear e recorrente, de acordo com os valores iniciais definidos, podendo ser atrasados ou adiantados.

Situação de validação: Os discentes devem perceber que a partir da matriz de Padovan, toda matriz geradora $\mathrm{Q}$ possui pelo menos duas propriedades, sendo elas: calcular o próximo termo, e guardar informações referente aos últimos termos da sequência. Para isso, é necessário a utilização de dois operadores: o operador principal, sendo aquele capaz de traduzir a forma como a sequência é calculada, e o operador de deslocamento, responsável por manter, excluir ou incluir novos elementos da sequência na matriz geradora $\mathrm{Q}$.

A segunda coluna da matriz geradora, representa o operador de deslocamento. A primeira linha da matriz representa os termos iniciais da sequência, escritos na forma do mais recente para o mais antigo. Considerando uma sequência $S$ com coeficientes $\beta_{0}, \beta_{1}, \ldots, \beta_{n}, \beta \in \mathrm{Z}$, em que $\mathrm{Z}$ representa o conjunto dos números inteiros, com fórmula de recorrência dada por: $S_{n}=\beta_{1} S_{n-1}+\beta_{2} S_{n-2}+\ldots+\beta_{n} S_{0}$, os alunos deverão diagnosticar que a matriz é dada por:

$Q=\left[\begin{array}{cc}\beta_{1} & {[I]} \\ \mathrm{M} & \mathrm{M} \\ \beta_{n} & 0\end{array}\right]$, sendo $\beta_{1}, \ldots, \beta_{n}$ os coeficientes da recorrência da sequência, [I] a matriz de bloco dada pela matriz identidade, 0 o vetor linha, ambos os vetores de tamanho $\mathrm{g}-1$, em que g representa a ordem da sequência.

Situação de institucionalização: $O$ professor entra em ação validando as construções realizadas pelos alunos, e revelando a intenção da atividade proposta. Com isso, o docente irá mostrar que com essa técnica de construção da forma matricial da sequência, é possível obter a matriz geradora de outras sequências, analisando a sua recorrência e os valores iniciais.

Situação-problema 2: De acordo com as matrizes válidas para a sequência de Padovan, vistas nos teoremas apresentados acima (Teorema 1, 2, 3, 4, 5 e 6), qual a regra de construção para a obtenção dessas matrizes, iniciando com uma matriz base? Quantas matrizes válidas existem para uma sequência linear e recorrente?

Situação de ação: Nessa fase inicial, os estudantes deverão analisar as matrizes de Padovan, e perceber como foram obtidas a partir da matriz base dada, levando em 
consideração os seus conhecimentos prévios referentes ao conteúdo de matrizes vistos em álgebra linear.

Situação de formulação: Durante essa etapa, os estudantes deverão perceber que ao permutar uma linha e consequentemente a coluna permutada, obtém-se uma matriz geradora de uma sequência linear. Para a obtenção do Teorema 2, realizando a permutação $L_{3}, L_{1}, L_{2}$, tem-se que:

$$
\left.\left.Q=\left[\begin{array}{lll}
0 & 1 & 0 \\
1 & 0 & 1 \\
1 & 0 & 0 \\
4 & 2 & 4 \\
C_{1} & C_{2} & C_{3}
\end{array}\right]\right\} \begin{array}{l}
L_{1} \\
L_{2} \\
L_{3}
\end{array} \rightarrow\left[\begin{array}{lll}
1 & 0 & 0 \\
0 & 1 & 0 \\
1 & 0 & 1 \\
4 & 2 & 4 \\
C_{1} & C_{2} & C_{3}
\end{array}\right]\right\} \begin{aligned}
& L_{3} \\
& L_{1} \\
& L_{2}
\end{aligned}
$$

Assim, permutando as colunas de acordo com as linhas permutadas anteriormente, tem-se que:

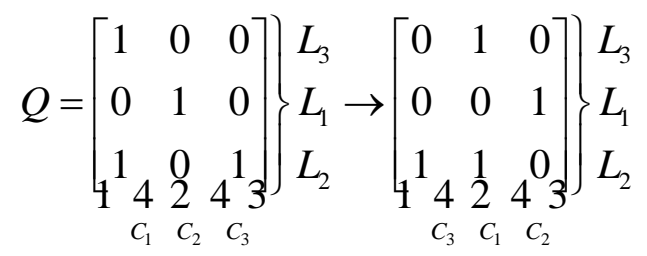

Obtendo a matriz do Teorema 2, onde realizando as mesmas permutações para a matriz elevada à $n$-ésima potência, obtêm-se os termos de Padovan.

Portando, as permutações das linhas e consequentemente das colunas das matrizes válidas da sequência de Padovan são:

$$
\begin{aligned}
& L_{1}, L_{2}, L_{3} \rightarrow C_{1}, C_{2}, C_{3}, \\
& L_{1}, L_{3}, L_{2} \rightarrow C_{1}, C_{3}, C_{2}, \\
& L_{2}, L_{1}, L_{3} \rightarrow C_{2}, C_{1}, C_{3}, \\
& L_{2}, L_{3}, L_{1} \rightarrow C_{2}, C_{3}, C_{1}, \\
& L_{3}, L_{1}, L_{2} \rightarrow C_{3}, C_{1}, C_{2}, \\
& L_{3}, L_{2}, L_{1} \rightarrow C_{3}, C_{2}, C_{1} .
\end{aligned}
$$

Obtendo assim as demais matrizes válidas. Para a sequência de Padovan existem seis matrizes, encontradas de acordo com a permutação de suas linhas e colunas, onde foram realizadas seis permutações, pois existem três linhas e três colunas na matriz, assim: $3 !=6$.

Situação de validação: Os estudantes deverão validar que para a obtenção de outras matrizes válidas de uma sequência linear e recorrente, é possível realizar permutações das linhas e posteriormente das colunas permutadas, e assim como aconteceu para a sequência de Padovan, acontece também para as outras sequências. 
De acordo com a permutação realizada entre linhas e colunas para a obtenção das outras matrizes válidas, sabendo que a ordem da matriz é de acordo com a ordem da sequência, temos que a quantidade de matrizes válidas varia de acordo com a ordem da sequência. Logo, para uma sequência de ordem 2, existem duas matrizes válidas (2!) de ordem 2x2, para uma sequência de ordem 3, existem seis matrizes válidas (3!) de ordem $3 \times 3$ e, para uma sequência de ordem n, existirão n! matrizes válidas de ordem nxn.

Situação de institucionalização: $O$ professor assume o papel principal da atividade, mostrando aos alunos se as discussões estão corretas, e caso contrário, analisa o erro encontrado pelos estudantes durante a aplicação.

Com essas duas atividades propostas conseguimos mostrar a regra de construção das matrizes geradoras da sequência de Padovan, podendo ser aplicadas a outras sequências lineares e recorrentes, não só de terceira ordem, mas também para outras ordens.

Para tornar ainda mais claro o ensino da forma matricial, podemos discutir a matriz de k-Pell, onde a sequência de k-Pell é uma sequência de segunda ordem com recorrência $P_{k, n}=2 P_{k, n-1}+k P_{k, n-2}, n \geq 2$ e $P_{k, 0}=0, P_{k, 1}=1$, existindo a matriz [3]:

$$
Q=\left[\begin{array}{ll}
2 & k \\
1 & 0
\end{array}\right]
$$

Assim, transformando essa matriz na matriz base, podemos reescrevê-la como:

$$
Q=\left[\begin{array}{ll}
2 & 1 \\
k & 0
\end{array}\right]
$$

Logo, na primeira coluna temos o operador, carregando os coeficientes da sequência de k-Pell, e na coluna temos o valor 1 e 0 , de acordo com a fórmula anteriormente discutida na atividade proposta. Com isso, ao elevarmos a $n$-ésima potência, teremos segundo Alves e Catarino [3]:

$$
Q^{n}=\left[\begin{array}{cc}
P_{k, n+1} & P_{k, n} \\
k P_{k, n} & k P_{k, n-1}
\end{array}\right]
$$

Concretizando a teoria discutida em que na primeira linha são apresentados os valores da sequência, de modo que nas linhas seguintes apareçam os termos anteriores ao primeiro termo inserido. 


\section{Conclusão}

Portanto, podemos perceber que foi possível, nesse trabalho, realizar um estudo e ensino da forma matricial de sequências lineares e recorrentes, sendo estruturado de forma à utilizar a metodologia de ensino baseada na Teoria das Situações Didáticas. Primordialmente, foi realizado um estudo sobre as sequências lineares e recorrentes, relatando ainda a problemática existente referente a construção das matrizes geradoras desses tipos de sequências. Seguindo esse processo, tomamos como exemplo as matrizes geradoras da Sequência de Padovan, para finalizarmos esse estudo.

Nessa perspectiva, definimos uma matriz de construção, denominada de matriz base, explicando a técnica do operador e a sua ligação com os coeficientes da fórmula de recorrência da sequência. Foi possível ainda verificar a variação da matriz base, possibilitando a obtenção de outras matrizes de acordo com a permutação de linhas e colunas. Destacamos ainda que esse conteúdo pode ser ensinado em cursos de formação inicial de professores, onde são estudadas sequências na disciplina de História da Matemática, visto que os livros referentes à essa disciplina e, em ementas de cursos, abordam tal assunto.

Por fim, estimula-se que o percurso abordado metodologicamente nesta pesquisa sirva como referência para sistematizar futuras pesquisas na área de ensino de matemática, com base na Teoria das Situações Didáticas. Além de sanar o problema encontrado diante artigos de matemática pura em que abordam a forma matricial sem realizar uma explicação de como foi alcançada determinada matriz e, a existência de outras para sequências lineares e recorrentes.

Como apresentado no trabalho de Alves [2], estimula-se ainda a utilização de recursos computacionais para o ensino de sequências lineares e recorrentes, visto que cada vez mais estão integrando ferramentas computacionais com o ensino.

\section{Agradecimentos}

A parte de desenvolvimento da pesquisa no Brasil contou com o apoio financeiro do Conselho Nacional de Desenvolvimento Cientifico e Tecnológico-CNPq e da Coordenação de Aperfeiçoamento de Pessoal de Nível Superior-CAPES. A parte de desenvolvimento da pesquisa em Portugal é financiado por Fundos Nacionais através da FCT-Fundação para a Ciência e a Tecnologia, I. P, no âmbito do projeto UID/CED/00194/2020. 


\section{Referências}

[1] ALMOULOUD, S. A. Fundamentos da didática da matemática. $1^{\text {a }}$ ed. Curitiba: Editora UFPR, v. 1, 2007.

[2] ALVES, F. R. V. Visualização de integrais impróprias em um parâmetro com o auxílio do Geogebra. TEAR: Revista de Educação, Ciência e Tecnologia, v. 3, n. 1, p. 1-15, 2014. Disponível em https://periodicos.ifrs.edu.br/index.php/tear/article/view/1821. Acesso em: 02 set, 2019.

[3] ALVES, F. R. V.; CATARINO, P. M. M. C. Sequência matricial generalizada de Fibonacci e sequência matricial k-Pell: propriedades matriciais. C.Q.D.-Revista Eletrônica Paulista de Matemática, v. 15, p. 39-54, 2019. Disponível em https://www.fc.unesp.br/\#!/departamentos/matematica/revista-cqd/. Acesso em: 13 out, 2019.

[4] BROUSSEAU, G. Perspective pour la didactique des mathématiques: vingt ans de didactique des mathématiques em France. Paris: La Pensée Sauvage, 1994.

[5] BROUSSEAU, G. Théorie des situations didactiques. Grenoble La Pensée Sauvage, 1998.

[6] OLIVEIRA, R. R.; ALVES, F. R. V. An investigation of the Bivariate Complex Fibonacci Polynomials supported in Didactic Engineering: an application of Theory of Didactics Situations (TSD). Acta Scientiae, v. 21, n. 3, p. 170-195, 2019. Disponível em http://www.periodicos.ulbra.br/index.php/acta/article/view/3940. Acesso em: 04 set, 2019.

[7] OLIVEIRA, R. R.; ALVES, F. R. V.; SILVA, R. S. O estudo de definições matemáticas no contexto de investigação histórica: um experimento didático envolvendo Engenharia Didática e sequências polinomiais de Fibonacci. TEAR: Revista de Educação, Ciência e Tecnologia, v. 6, n. 1, p. 1-14, 2017. Disponível em https://periodicos.ifrs.edu.br/index.php/tear/article/view/2108. Acesso em: 16 out, 2019.

[8] SANTOS, A. A.; ALVES, F. R. V. O estudo e o ensino da Sequência de Fibonacci numa abordagem atualizada. Revista Thema, v. 13, n. 2, p. 42-53, 2016. Disponível em http://revistathema.ifsul.edu.br/index.php/thema/article/viewFile/357/308. Acesso em: 10 out, 2019.

[9] SILVA, M. de O. P. da. As relações didático-pedagógicas no ensino de Geometria com o software Cabre Geometre. Curitiba, 2008.

[10] SEENUKUL, P.; et al. Matrices which have similar properties to padovan q matrix and its generalized relations. Sakon Nakhon Rajabhat University Journal of Science and Technology, v. 7, n. 2, p. 90-94, 2015. Disponível em https://www.tcithaijo.org/index.php/snru_journal/article/view/43656. Acesso em 10 set, 2019.

[11] SOKHUMA, Kritsana. Padovan q-matrix and the generalized relations. Applied Mathematical Sciences, v. 7, p. 2777-2780, 01 2013. Disponível em http://www.mhikari.com/ams/ams-2013/ams-53-56-2013/sokhumaAMS53-56-2013.pdf. Acesso em 09 set, 2019. 
[12] STEWART, I. Tales of a neglected number. Mathematical Recreations Scientific American, v. 274, n 6, p. 102-103, 1996. Disponível em https://www.scientificamerican.com/article/tales-of-a-neglected-number/. Acesso em 15 out, 2019.

[13] TEIXEIRA, P. J. M.; PASSOS, C. C. M. Um pouco da teoria das situações didáticas (tsd) de Guy Brousseau. Zetetiké, v. 21, n. 39, p.155-168, 2013. Disponível em https://www.fe.unicamp.br/revistas/ged/zetetike/article/view/4327/5110. Acesso em: 02 out, 2019.

[14] VIEIRA, R. P. M.; ALVES, F. R. V. A Sequência de Padovan e o número plástico: uma análise prévia e a priori. Research, Society and Development. v. 8, n. 8, p. 1-21, 2019. Disponível em https://rsd.unifei.edu.br/index.php/rsd/article/view/1212/988. Aceso em: 17 out, 2019.

[15] VOET, C.; SCHOONJANS, Y. Benidictine thought as a catalist for 20tm century liturgical space: the motivation behind dom hans van der laan $\mathrm{s}$ aesthetic church arquitectury. Proceeding of the 2 nd international conference of the Europa Architetural History of Network, p. 255-261, 2012. 